\title{
Testing discrete symmetries with neutral kaons at KLOE-2
}

\author{
Antonio Di Domenico* \\ on behalf of the KLOE-2 collaboration \\ Dipartimento di Fisica, Sapienza Università di Roma, and INFN Sezione di Roma, Rome, Italy \\ E-mail: antonio.didomenicodromal.infn.it
}

The KLOE-2 experiment at the INFN Laboratori Nazionali di Frascati has successfully concluded its data-taking at the DA $\Phi$ NE collider collecting an integrated luminosity of $5.5 \mathrm{fb}^{-1}$ at the $\phi$ resonance peak. Together with the data sample collected by its predecessor KLOE, the total integrated luminosity of $8 \mathrm{fb}^{-1}$ represents the largest existing data sample in the world collected at an $e^{+} e^{-}$ collider at the $\phi$ meson peak, corresponding to $\sim 2.4 \times 10^{10} \phi$-mesons produced. The entanglement in the neutral kaon pairs produced at the DAФNE $\phi$-factory is a unique tool to test discrete symmetries and quantum coherence at the utmost sensitivity, in particular strongly motivating the experimental searches of possible CPT violating effects, which would unambiguously signal New Physics. The lepton charge asymmetry measured in $\mathrm{K}_{\mathrm{S}}$ semileptonic decays with $1.63 \mathrm{fb}^{-1}$ of KLOE data, improving the statistical uncertainty of the present result by about a factor two, is reported, together with the tests of Time reversal and CPT symmetries in neutral kaon transition processes, and the search for the $\mathrm{CP}$ violating $\mathrm{K}_{\mathrm{S}} \rightarrow 3 \pi^{0}$ decay with the newly acquired KLOE-2 data.

The 39th International Conference on High Energy Physics (ICHEP2018)

4-11 July, 2018

Seoul, Korea

${ }^{*}$ Speaker. 


\section{Introduction}

DA $\Phi N E$, the Frascati $\phi$-factory, is an $e^{+} e^{-}$collider working at a center of mass energy of $\sqrt{s} \sim 1020 \mathrm{MeV}$ [1], corresponding to the peak of the $\phi$ resonance. The KLOE experiment at DAФNE completed its first data taking campaign in March 2006 with a total integrated luminosity of $\sim 2.5 \mathrm{fb}^{-1}$, corresponding to a production of $\sim 7.5 \times 10^{9} \phi$-mesons and $\sim 2.5 \times 10^{9} \mathrm{~K}^{0} \overline{\mathrm{K}}^{0}$ pairs.

After the KLOE run, DA $\Phi$ NE has been upgraded implementing an innovative collision scheme based on a crab-waist configuration [2,3]. The KLOE-2 experiment [4, 5], aiming to continue and extend the physics program of its predecessor, started its data-taking in November 2014 at the upgraded DAФNE, with an improved KLOE detector, and completed it by March 2018 after collecting a total integrated luminosity of $\sim 5.5 \mathrm{fb}^{-1}$. The KLOE-2 physics program has been described in detail in Ref. [4] and among the main issues includes neutral kaon interferometry and tests of discrete symmetries and quantum mechanics.

\section{Entangled neutral K mesons at KLOE-2}

At a $\phi$-factory neutral kaons are produced in the entangled state:

$$
|i\rangle=\frac{1}{\sqrt{2}}\left\{\left|\mathrm{~K}^{0}\right\rangle\left|\overline{\mathrm{K}}^{0}\right\rangle-\left|\overline{\mathrm{K}}^{0}\right\rangle\left|\mathrm{K}^{0}\right\rangle\right\}=\frac{\mathscr{N}}{\sqrt{2}}\left\{\left|\mathrm{~K}_{\mathrm{S}}\right\rangle\left|\mathrm{K}_{\mathrm{L}}\right\rangle-\left|\mathrm{K}_{\mathrm{L}}\right\rangle\left|\mathrm{K}_{\mathrm{S}}\right\rangle\right\}
$$

with $\mathscr{N} \simeq 1$ a normalization factor.

The observable quantity is the double differential decay rate of the state $|i\rangle$ into decay products $f_{1}$ and $f_{2}$ at proper times $t_{1}$ and $t_{2}$, respectively. After integration on $\left(t_{1}+t_{2}\right)$ at fixed time difference $\Delta t=t_{1}-t_{2}$, the decay intensity can be written as follows [6]:

$$
I\left(f_{1}, f_{2} ; \Delta t\right)=C_{12}\left\{\left|\eta_{1}\right|^{2} e^{-\Gamma_{L} \Delta t}+\left|\eta_{2}\right|^{2} e^{-\Gamma_{S} \Delta t}-2\left|\eta_{1}\right|\left|\eta_{2}\right| e^{-\frac{\left(\Gamma_{S}+\Gamma_{L}\right)}{2} \Delta t} \cos \left[\Delta m \Delta t+\phi_{2}-\phi_{1}\right]\right\}
$$

with $\Delta m=m_{L}-m_{S}$, and

$$
\eta_{i} \equiv\left|\eta_{i}\right| e^{i \phi_{i}}=\frac{\left\langle f_{i}|T| \mathrm{K}_{\mathrm{L}}\right\rangle}{\left\langle f_{i}|T| \mathrm{K}_{\mathrm{S}}\right\rangle} ; C_{12}=\frac{|\mathscr{N}|^{2}}{2\left(\Gamma_{S}+\Gamma_{L}\right)}\left|\left\langle f_{1}|T| \mathrm{K}_{\mathrm{S}}\right\rangle\left\langle f_{2}|T| \mathrm{K}_{\mathrm{S}}\right\rangle\right|^{2}
$$

Expression (2.2) is valid for $\Delta t \geq 0$, while for $\Delta t<0$ the substitutions $\Delta t \rightarrow|\Delta t|$ and $1 \leftrightarrow 2$ have to be applied.

\section{Measurement of $K_{S}$ semileptonic charge asymmetry}

At KLOE a $\mathrm{K}_{\mathrm{S}}$ is tagged by identifying the interaction of the $\mathrm{K}_{\mathrm{L}}$ in the calorimeter $\left(\mathrm{K}_{\mathrm{L}}{ }^{-}\right.$ crash). In fact about $50 \%$ of the produced $\mathrm{K}_{\mathrm{L}}$ 's in $\phi \rightarrow \mathrm{K}_{\mathrm{S}} \mathrm{K}_{\mathrm{L}}$ events reach the calorimeter before decaying; their associated interactions are identified by a high energy, neutral and delayed deposit in the calorimeter, i.e. not associated to any charged track in the event, and delayed of $\sim 30 \mathrm{~ns}$ (as $\beta_{K} \sim 0.22$ ) with respect to a photon coming from the interaction region. Pure $\mathrm{K}_{\mathrm{S}}$ samples have been selected exploiting this tagging technique. In particular $\mathrm{K}_{\mathrm{S}} \rightarrow \pi e v$ decays are selected requiring a $\mathrm{K}_{\mathrm{L}}$-crash and two tracks forming a vertex close to the IP, and associated with two energy deposits 
in the calorimeter. Pions and electrons are recognized using their TOF. A control sample of $\phi \rightarrow$ $\mathrm{K}_{\mathrm{S}} \mathrm{K}_{\mathrm{L}} \rightarrow \pi^{0} \pi^{0}, \pi e v$ with the semileptonic decay close to the IP has been used to correct efficiencies evaluated with Monte Carlo. The analysis of a KLOE data sample corresponding to $1.63 \mathrm{fb}^{-1}$ yields a measurement of the $\mathrm{K}_{\mathrm{S}}$ semileptonic charge asymmetry [7]: $A_{S}(-4.9 \pm 5.7 \pm 2.6) \times 10^{-3}$, improving by about a factor two the statistical uncertainty with respect to the previous KLOE result [8]. The combination of the two measurements yields:

$$
A_{S}=(-3.8 \pm 5.0 \pm 2.6) \times 10^{-3} .
$$

The measured $A_{S}$ value (3.1) is consistent with the expectation $A_{S}=A_{L} \simeq 3.3 \times 10^{-3}$ imposed by CPT invariance $\left(A_{L}\right.$ is the $\mathrm{K}_{\mathrm{L}}$ semileptonic charge asymmetry and is precisely measured [9]), while its uncertainty is approaching the level necessary to reveal $\mathrm{CP}$ violation in the $\mathrm{K}_{\mathrm{S}}$ (i.e. $A_{S} \neq$ 0 ). From the sum and the difference of $A_{S}$ and $A_{L}$ one can perform a CPT test. In fact using the values of $A_{L}, \Re \delta$, and $\Re \varepsilon^{1}$ from other experiments [9], the real part of the CPT violating and $\Delta S=\Delta Q$ violating (conserving) parameter $x_{-}(y)$ in semileptonic decay amplitudes can be evaluated [7]: $\Re x_{-}=\frac{A_{S}-A_{L}}{4}-\Re \delta=(-2.0 \pm 1.4) \times 10^{-3}, \Re y=\Re \varepsilon-\frac{A_{S}+A_{L}}{4}=(1.7 \pm 1.4) \times 10^{-3}$, improving the uncertainty with respect to previous results. The uncertainty on $A_{S}$ can be further reduced at the level of $\approx 3 \times 10^{-3}$ with the analysis of the full KLOE-2 data sample.

\section{Test of Time reversal and CPT symmetries in neutral kaon transitions}

As explained in Refs [10,11], exploiting the entanglement of the neutral kaon pair in the initial state (2.1), it is possible to exchange in and out states in a transition process. For instance it is possible to observe the transition $\mathrm{K}^{0} \rightarrow \mathrm{K}_{-}$and its T-conjugated $\mathrm{K}_{-} \rightarrow \mathrm{K}^{0}$ or CPT-conjugated $\mathrm{K}_{-} \rightarrow \overline{\mathrm{K}}^{0}$ process, with $\mathrm{K}_{-}$defined as the state filtered by the decay into $3 \pi^{0}$, a pure $\mathrm{CP}=-1$ final state $^{2}$. Any deviation from unity of the corresponding ratios of probabilities

$$
\begin{aligned}
R_{2, \mathrm{~T}}(\Delta t) & =P\left[\mathrm{~K}^{0}(0) \rightarrow \mathrm{K}_{-}(\Delta t)\right] / P\left[\mathrm{~K}_{-}(0) \rightarrow \mathrm{K}^{0}(\Delta t)\right] \\
R_{2, \mathrm{CPT}}(\Delta t) & =P\left[\mathrm{~K}^{0}(0) \rightarrow \mathrm{K}_{-}(\Delta t)\right] / P\left[\mathrm{~K}_{-}(0) \rightarrow \overline{\mathrm{K}}^{0}(\Delta t)\right]
\end{aligned}
$$

would be an unambiguous signal of $\mathrm{T}$ or CPT violation, respectively. In this case the relevant observable quantities at a $\phi$-factory are the ratios of double decay intensities ${ }^{3}$ :

$$
R_{2, \mathrm{~T}}^{\exp }(\Delta t) \equiv \frac{I\left(\ell^{-}, 3 \pi^{0} ; \Delta t\right)}{I\left(\pi \pi, \ell^{+} ; \Delta t\right)} ; R_{2, \mathrm{CPT}}^{\exp }(\Delta t) \equiv \frac{I\left(\ell^{-}, 3 \pi^{0} ; \Delta t\right)}{I\left(\pi \pi, \ell^{-} ; \Delta t\right)} .
$$

In particular the observable double ratio:

$$
D R_{C P T} \equiv \frac{R_{2, C P T}^{\exp }\left(\Delta t \gg \tau_{S}\right)}{R_{4, C P T}^{\exp }\left(\Delta t \gg \tau_{S}\right)}=1-8 \Re \delta-8 \Re x_{-},
$$

with $R_{4, \mathrm{CPT}}^{\exp }(\Delta t) \equiv \frac{I\left(\ell^{+}, 3 \pi^{0} ; \Delta t\right)}{I\left(\pi \pi, \ell^{+} ; \Delta t\right)}$, is predicted to be strictly equal to unity by CPT invariance, and constitutes one of the most robust and cleanest observable for a model independent CPT test that has never been performed in the neutral kaon system.

\footnotetext{
${ }^{1} \varepsilon$ and $\delta$ are the usual complex parameters describing $C P$ and $C P T$ violation in the mixing of neutral kaons.

${ }^{2} \mathrm{~K}_{-}$is assumed to be orthogonal to $\mathrm{K}_{+}$, the state filtered by the decay into $\pi \pi$, a pure $\mathrm{CP}=+1$ final state.

${ }^{3}$ In the following the semileptonic decays $\pi^{+} \ell^{-} v$ and $\pi^{-} \ell^{+} \bar{v}$ will be denoted for brevity as $\ell^{-}$and $\ell^{+}$, respectively.
} 
The analyses of KLOE and KLOE-2 data are ongoing and at an advanced stage, allowing to achieve a precision $<1 \%$ on the relevant $\mathrm{T}$ and CPT observables. Noting that there exist a connection between $D R_{C P T}$ and $A_{S, L}$, i.e. $D R_{C P T}=1+2\left(A_{L}-A_{S}\right)$ [11], a first preliminary CPT test in transitions is obtained: $D R_{C P T}=1.016 \pm 0.011$, consistent with CPT invariance.

\section{Search for the $\mathrm{CP}$-violating $\mathrm{K}_{\mathrm{S}} \rightarrow 3 \pi^{0}$ decay}

The Standard Model prediction for the branching ratio of the CP-violating decay $\mathrm{K}_{\mathrm{S}} \rightarrow 3 \pi^{0}$ is $\mathrm{BR}\left(\mathrm{K}_{\mathrm{S}} \rightarrow 3 \pi^{0}\right) \sim 1.9 \times 10^{-9}$, making the direct observation of this decay quite a challenge. The best upper limit comes from the analysis of $1.7 \mathrm{fb}^{-1}$ collected by KLOE, searching for six photons coming from the IP and a $\mathrm{K}_{\mathrm{L}}$-crash [12]: $\mathrm{BR}\left(\mathrm{K}_{\mathrm{S}} \rightarrow 3 \pi^{0}\right)<2.6 \times 10^{-8}$ at $90 \%$ C.L. . This result can be further improved with the analysis of the additional $5.5 \mathrm{fb}^{-1}$ of data collected by KLOE2. A preliminary analysis of $300 \mathrm{pb}^{-1}$ shows the good quality of KLOE-2 data even in presence of a larger machine background with respect to KLOE. After hardening the selection criteria to get about ten times better background rejection, and after applying the scheme of the previous analysis [12], only one candidate event survives, leaving room to improvements and the possibility to reach a final sensitivity on the BR below $10^{-8}$.

\section{Conclusions}

The entangled neutral kaon system at DAФNE is an excellent laboratory for the study of discrete symmetries. The KLOE-2 experiment at the upgraded DA $\Phi$ NE successfully completed its data taking campaign collecting $L=5.5 \mathrm{fb}^{-1}$ by the end of March 2018. The whole KLOE and KLOE-2 data sample $\left(8 \mathrm{fb}^{-1}\right)$ is worldwide unique for typology and statistical relevance and its analysis is in progress. The study of discrete symmetries with neutral kaons is one of the key issues at KLOE-2, and the precision of several tests performed by KLOE will be further improved.

\section{References}

[1] A. Gallo et al.. DAFNE status report, Conf.Proc. C060626 (2006) 604-606, SLAC-PUB-12093.

[2] M. Zobov et al., Phys. Rev. Lett. 104, 174801 (2010).

[3] C. Milardi et al., JINST 7, 2012,T03002.

[4] G. Amelino-Camelia et al., Eur. Phys. J. C 68, 619 (2010).

[5] P. Gauzzi, these proceedings

[6] A. Di Domenico et al., Handbook on Neutral Kaon Interferometry at a $\phi$-factory, Frascati Phys. Ser. 43 (2007).

[7] A. Anastasi et al., KLOE-2 collaboration, JHEP 09, 139 (2018).

[8] F. Ambrosino et al., KLOE collaboration, Phys. Lett. B 636 (2006) 173.

[9] C. Patrignani et al. (Particle Data Group), Chin. Phys. C 40, 100001 (2016).

[10] J. Bernabeu, A. Di Domenico, P. Villanueva-Perez, Nucl. Phys. B. 868, 102 (2013)

[11] J. Bernabeu, A. Di Domenico, P. Villanueva-Perez, JHEP 10, 139 (2015)

[12] D. Babusci et al., KLOE-2 collaboration, Phys. Lett. B 723, 54 (2013). 